Literature Reviews

\title{
Potential Pathomechanism of Neuro-COVID-19
}

\author{
${ }^{1,3}$ Abir Fguirouche, ${ }^{2}$ Nissrine Louhab, ${ }^{2}$ Mohamed Chraa, ${ }^{2}$ Najib Kissani and ${ }^{3,4}$ Brahim Admou \\ ${ }^{1}$ Faculty of Sciences Semlalia, Cadi Ayyad University, Marrakech, Morocco \\ ${ }^{2}$ Department of Neurology, University Hospital Mohamed VI, Cadi Ayyad University, Marrakech, Morocco \\ ${ }^{3}$ Department of Immunology, Center of Clinical Research, University Hospital Mohamed VI, Marrakech, Morocco \\ ${ }^{4}$ B2S Research Laboratory, Faculty of Medicine and Pharmacy, Cadi Ayyad University, Marrakech, Morocco
}

\author{
Article history \\ Received: 06-10-2020 \\ Revised: 05-12-2020 \\ Accepted: 07-12-2020 \\ Corresponding Author: \\ Fguirouche Abir \\ Faculty of Sciences Semlalia, \\ Cadi Ayyad University, \\ Marrakech, Morocco \\ E-mail: fgrabir@gmail.com
}

\begin{abstract}
Gathered data about SARS-CoV-2 have shown strong evidence in terms of neuroinvasive and neurotropic capacities of the virus to reach and cause eventual damage to the Central Nervous System. The spread of the virus may occur through direct and indirect ways, leading to anosmia and/or ageusia. These signs have been observed in many COVID-19 patients and are then included in the diagnostic criteria for Neuro-COVID19. The invasion of SARS-CoV-2 into the dorsal vagal complex of the brainstem has a harmful impact on the respiratory rhythm, with even respiratory failure. There is evidence that immune response abnormalities including cytokine storm and neuro-inflammation influence not only the disease severity, but also the occurrence of neurological and neurodegenerative manifestations. Many umbrella zones remain unclear about Neuro-COVID-19, which requires more consideration in its clinical, biological and therapeutic aspects and may open new research perspectives.
\end{abstract}

Keywords: Sars-CoV-2, COVID-19, Nervous System, Pathomechanisms, Cytokine Storm

\section{Introduction}

For the first time in December 2019, Wuhan in China knew an outbreak of Severe Acute Respiratory Syndrome Coronavirus 2 (SARS-CoV-2) also known as Corona Virus Disease 2019 (COVID-19) (Thompson, 2020). In addition to systemic and respiratory symptoms researchers found that many COVID-19 patients develop neurological manifestations, such as headache, febrile seizure, encephalitis, dizziness, ataxia, disturbed consciousness and paresthesia (Mao et al., 2020). These clinical manifestations occur more likely in severely affected patients than those with mild or moderate disease (Mao et al., 2020). Many studies suggest that the nervous system involvement may be due to the neuroinvasive and neurotropic capabilities of SARSCoV-2 (Giraudon and Bernard, 2010). The spread from the respiratory tract into the Central Nervous System (CNS) is done through many potential ways, especially the olfactory tract, fact that needs more enlightenment (Talbot et al., 2007).

The aim of this review is to emphasize the potential pathomechanisms of the nervous system involvement during COVID-19.

\section{SARS-CoV-2 and Receptors: ACE2, TMPRSS2}

Coronaviruses (CoVs) are non-segmented positivesense RNA viruses with neurotropic and neuroinvasive capacities. They can infect many different species and are responsible for respiratory and enteric disorders (Vabret et al., 2009; Buchmeier and Lane, 1999; Cavanagh, 2005).

SARS-CoV-2 can bind to the Angiotensin-Converting Enzyme 2 (ACE2) receptor in humans ( $\mathrm{Lu}$ et al., 2020; Zhang et al., 2020), which has previously been identified as the functional cellular receptor for other SARS-CoVs (Li et al., 2003), with a polymorphic expression, particularly on alveolar epithelial cells, intestinal enterocytes, arterial and venous endothelial cells (Hamming et al., 2004). In the brain, the implication of ACE2 is still not well documented since the expression of its receptor was first found only in brain blood vessels, but other recent studies show that ACE2 and its receptors can also be found in CNS glial cells and sustentacular cells of the olfactory epithelium, which indicates that human brain is a potential target of COVID-19 (Fig. 1) (Xia and Lazartigues, 2010; Baig et al., 2020; Bilinska et al., 2020). 


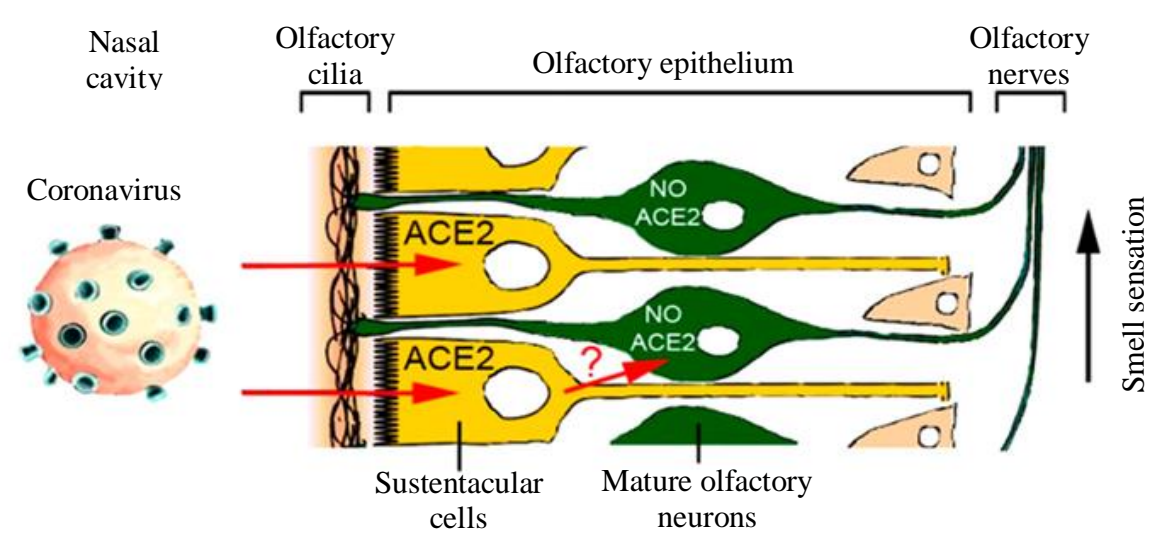

Fig. 1: Schematic representation showing the postulated entry of SARS-CoV-2 into the Nervous system through its binding to ACE2 receptors in sustentacular cells of the Nasal cavity (Bilinska et al., 2020)

In a recent study, SARS-CoV-2 invasion was confirmed to occur through its obligatory binding to the ACE2 receptor, while the viral uptake is facilitated by the priming protease, TMPRSS2. High expression of ACE2 and TMPRSS2 increases the virus binding capacity and susceptibility to affect the sustentacular cells of the olfactory epithelium, thus indicating that these olfactory cells may be targeted by the virus (Bilinska et al., 2020; Hoffmann et al., 2020; Sungnak et al., 2020).

\section{Neurologic Manifestations}

Neurologic disorders of COVID-19 were first reported on February 19th, 2020 in a Chinese study with 214 patients, who displayed various neurologic manifestations in $36.4 \%$ of cases, including central and peripheral disorders, associated to skeletal muscle involvement (Mao et al., 2020). The occurrence of neurologic manifestations was noticed in patients with severe COVID-19, particularly acute cerebrovascular disease, altered consciousness, delirium, headache, skeletal muscle signs, Guillain-Barré syndrome and transverse myelitis (Mao et al., 2020; Ahmad and Rathore, 2020). Further, (Li et al., 2020a) conducted a study on 221 COVID-19 patients and described acute ischemic stroke, cerebral venous sinus thrombosis and cerebral hemorrhage in $5,0.5$ and $0.5 \%$ of cases respectively. A recent French study also reported neurologic manifestations in 58 of 64 patients with COVID-19, including encephalopathy, prominent agitation, confusion and corticospinal tract signs (Helms et al., 2020).

Coronavirus infections have been related to many other nervous system disorders, like infectious toxic encephalopathy as a concrete example characterizing Coronaviruses associated with acute viral respiratory infections. Patients with COVID-19 often suffer from severe hypoxia and viremia (Guo et al., 2020), which has the potential to cause toxic encephalopathy. Through a meta-analysis, (Tong et al., 2020) reported $52.73 \%$ of olfactory dysfunction among ten studies including 1627 COVID-19 cases, while nine other studies showed $43.93 \%$ of gustatory dysfunction among 1390 of COVID-19 patients. The cause of the olfactory and the gustatory disturbance has not yet been definitively identified (Tong et al., 2020; Soler et al., 2020).

\section{Pathomechanism}

It was proven that most viruses belonging to CoVs family share the similar structure and infection pathways, therefore the mechanisms previously described for other CoVs may also apply to SARS-CoV-2 (Vabret et al., 2009; Buchmeier and Lane, 1999).

\section{Neuroinvasive and Neurotropic Capacities of SARS-CoV-2}

Experimental studies conducted on other coronaviruses suggest wider tissue invasiveness and evident neurotropism as a current hypothesis, which may impact the pathogenesis of the infection and results in complex clinical scenarios (Ding et al., 2004). Data collected from these studies demonstrated that $\beta$ coronaviruses to which SARS-CoV-2 belongs are not only present in the respiratory tract but also invade the CNS and infect the neurons. This has convincingly been proven in the case of the SARS-CoV, MERS$\mathrm{CoV}$ and the one responsible for porcine haemagglutinating encephalomyelitis (Dubé et al., 2018; Talbot et al., 1994).

Mao et al. (2020) reported that some patients develop COVID-19 related symptoms only after exhibiting neurologic manifestations. In the province of Beijing, a viral encephalitis case caused by SARS-COV-2 was confirmed by the presence of the virus in the cerebrospinal fluid and by the viral genome sequencing, supporting the theory that the new virus can reach and 
damage the nervous system (Xiang et al., 2020). In some other patients with severe symptoms of COVID-19, it is therefore likely that other pathogenic bacteria play a crucial role in the breakdown of the Blood-Brain Barrier (BBB). Also, secondary intracranial infections may cause headaches, projectile vomiting, vision loss and convulsions of the limbs. (Wu et al., 2020).

Current studies conducted on the impact of $\beta$-CoVs on the human organism in general and the nervous system in particular, suggest that the SARS-CoV-2 viral strain could infect the CNS, which had been demonstrated with other $\beta$-CoVs. In an animal model, cell culture studies have shown that neural cell lines as well as primary neurons are highly infected in the case of NCoVOC43, which is also responsible for encephalitis, associated with neural apoptosis and necrosis (Jacomy et al., 2006).

\section{Possible Pathophysiology of the Nervous System Involvement}

Coronaviruses are neuroinvasive and may enter the CNS through several ways. Recent hypothesis suggests that SARS-CoV-2 uses direct and indirect pathways to reach and affect the CNS components (Wu et al., 2020). Therefore, it is crucial to investigate these two major routes.

\section{a. Postulated Direct Pathways}

Proteins and genetic material from different viruses have been detected in various tissue samples from the nervous system, such as cerebrospinal fluid or the brain, suggesting that viruses can directly reach the nervous system and cause damage. Potentially as human CoVs, SARS-CoV-2 acts in the same way on the nervous system although more studies are needed to fully understand the mechanisms of the viral spread toward the nervous system (Koyuncu et al., 2013; Leber et al., 2016).

\section{a.1. The Haematogenous Way}

There is a rare evidence demonstrating the spread of SARS-CoV-2 to the nervous system through the bloodstream (Koyuncu et al., 2013; Desforges et al., 2020). As previously mentioned, entry of SARS-CoV-2 into host cells is mediated by ACE2 in a similar manner to SARS-CoV, while MERS-CoV binds to DipeptidylPeptidase 4 (DPP4) (Hoffmann et al., 2020; Raj et al., 2013). Using SARS-CoV-infected transgenic mice model of ACE2, experiments reported that the olfactory nerve was the direct pathway used by the virus to reach the brain. However, the presence of a number of infected sites indirectly linked to the olfactory bulb suggests the existence of non-neuronal pathways such as the hematogenous way (Netland et al., 2008). Another experimental animal model study carried out on mice infected with SARS-CoV, showed a disrupted barrier functions as well as downregulation of the ACE2 protein expression, which increases vascular permeability (Imai et al., 2008). In the same context, SARS-CoV-2 not only causes endothelial damage and entry into the bloodstream after binding to the ACE2 receptor on alveolar epithelial cells, but also infects epithelial cells and circulating immune cells. These infected immune cells, including leukocytes, spread easily in the circulatory system and transport the virus to other organs, causing fever, myalgia, fatigue, kidney failure and disorder of both CNS and PNS (Peripheral Nervous System). (Li et al., 2020b). The endothelial cells of the Blood Brain Barrier (BBB) are also potentially infected directly by the virus in the plasma, but the mechanism remains unknown (Huang et al., 2020).

\section{a.2. The Peripheral Nervous Way}

In postmortem studies, particles of SARS-CoV were found in the brain and displayed a cellular selectivity in neurons rather than glia (Ding et al., 2004; Xu et al., 2005). SARS-CoV-2 may invade the CNS via neuronal pathway by infecting sensory or motor nerve terminals, where it replicates, then uses a retrograde way to the soma, causing disruption of certain nuclei or neural circuits. This has been proven by numerous studies conducted on two other CoV families: MERS-CoV and SARS-CoV, which share homologous sequences with SARS-CoV-2 (Glass et al., 2004; Dubé et al., 2018).

The nasal epithelium and the CNS are channeled to the nasal cavity and the forebrain thanks to the unique anatomical organization of the olfactory nerves and the olfactory bulb in the nasal cavity (Koyuncu et al., 2013). In an animal model, Bohmwald et al. (2018) reported a restriction of $\mathrm{CoV}$ invasion in the CNS following removal of the olfactory bulb. As a potential route for CNS involvement by the virus, olfactory neuronal transport is a concrete example of a neural pathway, which would be useful for understanding the loss of smell, taste and vision in some patients with COVID-19. Experimental studies on MERS-CoV and SARS-CoV served as evidence to conclude that once SARS-CoV-2 is attached to the nasal cavity mucosa, the sensory neurons of the olfactory epithelium maybe directly infected by the virus which is then transported into the CNS through the olfactory nerve (Li et al., 2016; Lochhead and Thorne, 2012). The trigeminal nerve can also be affected by the viral adhesion to the mucosa (Lochhead et al., 2019; Lochhead and Thorne, 2012). In humans, the cranial nerves responsible for the sense of taste are the vagus nerve (X), the facial nerve (VII) and the glossopharyngeal nerve (IX). Interestingly, the nucleus of the solitary tract in Medulla Oblongata and the thalamus are also involved. The loss of taste is basically due to an injury to one of the three cranial nerves, the solitary nuclei or the thalamus. Also, the loss of appetite in Covid-19 patients and respiratory failure are related to infection of the nuclei of the solitary tract 
and thalamus, both belonging to the Dorsal Vagal Complex (DVC), which control many autonomic activities, including breathing and food intake (Baig et al., 2020; Li et al., 2020c). Finally, about the loss of vision, more studies are required to establish the presence of a direct infection of the optic nerve since only one patient with conjunctivitis showed the presence of the viral genetic material in an ocular discharge (Sun et al., 2020).

\section{b. Postulated Indirect Mechanisms}

\section{b.1. Cytokine Dysregulation}

Human Coronaviruses ( $\mathrm{HCoV}$ ) causing severe pneumonia are associated with cytokine storm also known as induced hypercytokinemia, especially in immunocompetent individuals. This cytokine storm is characterized not only by an overproduction of more than 150 inflammatory cytokines and chemokines, but also a hyperactivation of $\mathrm{T}$ cells, macrophages and natural killer cells (Osterholm, 2005; Teijaro et al., 2014).

In general, viral infections are characterized by lung epithelial and endothelial cell apoptosis due to an important uncontrolled release of pro-inflammatory cytokines and chemokines such as IL-6, IL-8, IL-1 $\beta$, GM-CSF, CCL2, CCL-5, IP-10 and CCL3. Together in the presence of Reactive Oxygen Species (ROS), these factors are responsible for the Acute Respiratory Distress Syndrome (ARDS) (Reghunathan et al., 2005). Deceased SARS-CoV patients showed high serum levels of IFN- $\gamma$, IL-1, IL-6, IL-12, TGF $\beta$, CCL2, CXCL10, CXCL9, compared to patients with mild to moderate SARS-CoV (Chien et al., 2006), while severe infected MERS-CoV patients showed high levels of IL- 6 , IFN- $\alpha$, IL-8, CXCL-10 and CCL5 (Min et al., 2016). In the case of the new SARS-CoV-2, significant high levels of IL-2, IL-7, IL-10, GSCF, IP10, MCP1, MIP1a and TNF- $\alpha$ were found in sera of severely infected patients (Huang et al., 2020). Moreover, T cell Hyperactivation contributing to the severe injuries is due to the presence of high concentrations of CCR4+ CCR6+ Th17+ CD4T cells (Xu et al., 2020). Knowing that the cytokine storm can cause either acute or subacute CNS damages, a recent study reported the case of a COVID-19 woman who presented an acute necrotizing hemorrhagic encephalopathy and deceased after several days. This was explained as a rare condition associated with intracranial cytokine storm and $\mathrm{BBB}$ disruption. Peripheral inflammation releasing many cytokines may increase the permeability of the BBB providing a pathway for the virus to enter the brain (Poyiadji et al., 2020).

\section{b.2. Neuro-Inflammation and Neurological Disorders}

Currently, the evidence suggests that once in the CNS, SARS-CoV-2 may infect astrocytes and microglia and leads to a chronic neuro-inflammation, which is involved in the pathogenesis of a wide range of neurological disorders like Multiple Sclerosis (MS), Parkinson's Disease, Alzheimer's disease, Amyotrophic lateral sclerosis, Huntington disease, immune-mediated or post infectious encephalitis, cerebrovascular disease and peripheral nervous system disorders through the release of cytokines, ROS and other inflammatory mediators (Frank-Cannon et al., 2009). Cytokine Storm associated to typical SARS infection usually involves immune mechanisms leading to neurodegenerative diseases. Nevertheless, the immune response as well as the severity of SARS-CoV-2 infection varies depending on the age of patients. In general, elderly patients display severe clinical forms due to the decreased efficiency of the innate immune response (Boe et al., 2017).

Many studies have showed that cytokine trafficking across the BBB can cause acute necrotizing encephalopathy (Mehta et al., 2020; Poyiadji et al., 2020). A case of fatal Guillain-Barré Syndrome (GBS) related to COVID-19 has been reported, nonetheless, the circumstances of its development are not yet well established (Zhao et al., 2020). It has also been shown that neuroinflammation plays a major role in the mechanisms of psychiatric disorders since it induces changes in neurotransmitter metabolism and deregulation of the hypothalamic-pituitary-adrenal axis, activates microglia, alters neuroplasticity and triggers structural and functional changes in the brain. This could affect cognitive and behavioral abilities (Rhie et al., 2020).

Neuro-inflammation can be secondary to microglial and astrocyte activation in the case of epileptic syndromes through the release of some inflammatory cytokines such as IL-1 $\beta$, IL- 6 and TNF $\alpha$ in different cortical regions like the hippocampus and the neocortex (Reddy and Kuruba, 2013).

Finally, further studies investigating the effects of SARS-CoV-2 infection on the development and progression of neurologic and neuropsychiatric disorders are still required.

\section{Conclusion}

Human coronaviruses are neuroinvasive and neurotropic, their presence in the CNS is admitted as a fact. It is now believed that some COVID-19 patients develop Systemic Inflammatory Response Syndrome (SIRS) characterized by an uncontrolled release of inflammatory mediators which unfortunately may increase mortality. Data about the immune and inflammatory status of deceased patients with SARS-CoV-2 are emerging, which requires more experimentation studies to establish the exact mechanisms and consequences of COVID-19 on the nervous system and develop appropriate therapeutic strategies. 


\section{Author's Contributions}

Abir Fguirouche: Wrote the first draft of the manuscript, with bibliography search and management.

Nissrine Louhab: Contributed to writing and editing the manuscript.

Mohamed Chraa: Validated the clinical aspects of the manuscript.

Najib Kissani: Made a critical review and validated the manuscript.

Brahim Admou: Conceptualized, revised and approved the final version of the manuscript.

\section{Conflict of Interest Disclosure}

This review was conducted in the absence of any financial relationship that could be interpreted as a potential conflict of interest.

\section{References}

Ahmad, I., \& Rathore, F. A. (2020). Neurological manifestations and complications of COVID-19: A Literature review. Journal of Clinical Neuroscience.

Baig, A. M., Khaleeq, A., Ali, U., \& Syeda, H. (2020). Evidence of the COVID-19 virus targeting the CNS: Tissue distribution, host-virus interaction and proposed neurotropic mechanisms. ACS chemical neuroscience, 11(7), 995-998.

Bilinska, K., Jakubowska, P., Von Bartheld, C. S., \& Butowt, R. (2020). Expression of the SARS-CoV-2 Entry Proteins, ACE2 and TMPRSS2, in Cells of the Olfactory Epithelium: Identification of Cell Types and Trends with Age. ACS Chemical Neuroscience.

Boe, D. M., Boule, L. A., \& Kovacs, E. J. (2017). Innate immune responses in the ageing lung. Clinical \& Experimental Immunology, 187(1), 16-25.

Bohmwald, K., Galvez, N., Ríos, M., \& Kalergis, A. M. (2018). Neurologic alterations due to respiratory virus infections. Frontiers in cellular neuroscience, 12, 386.

Buchmeier, M. J., \& Lane, T. E. (1999). Viral-induced neurodegenerative disease. Current opinion in microbiology, 2(4), 398-402.

Cavanagh, D. (2005). Coronaviruses in poultry and other birds. Avian pathology, 34(6), 439-448.

Chien, J. Y., Hsueh, P. R., Cheng, W. C., Yu, C. J., \& Yang, P. C. (2006). Temporal changes in cytokine/chemokine profiles and pulmonary involvement in severe acute respiratory syndrome. Respirology, 11(6), 715-722.

Desforges, M., Le Coupanec, A., Dubeau, P., Bourgouin, A., Lajoie, L., Dubé, M., \& Talbot, P. J. (2020). Human coronaviruses and other respiratory viruses: underestimated opportunistic pathogens of the central nervous system?. Viruses, 12(1), 14.
Ding, Y., He, L. I., Zhang, Q., Huang, Z., Che, X., Hou, J. \& Geng, J. (2004). Organ distribution of severe acute respiratory syndrome (SARS) associated coronavirus (SARS-CoV) in SARS patients: implications for pathogenesis and virus transmission pathways. The Journal of Pathology: A Journal of the Pathological Society of Great Britain and Ireland, 203(2), 622-630.

Dubé, M., Le Coupanec, A., Wong, A. H., Rini, J. M., Desforges, M., \& Talbot, P. J. (2018). Axonal transport enables neuron-to-neuron propagation of human coronavirus OC43. Journal of virology, 92(17).

Frank-Cannon, T. C., Alto, L. T., McAlpine, F. E., \& Tansey, M. G. (2009). Does neuroinflammation fan the flame in neurodegenerative diseases ? Molecular neurodegeneration, 4(1), 1-13.

Giraudon, P., \& Bernard, A. (2010). Inflammation in neuroviral diseases. Journal of neural transmission, 117(8), 899-906.

Glass, W. G., Subbarao, K., Murphy, B., \& Murphy, P. M. (2004). Mechanisms of host defense following severe acute respiratory syndrome-coronavirus (SARS-CoV) pulmonary infection of mice. The Journal of Immunology, 173(6), 4030-4039.

Guo, Y. R., Cao, Q. D., Hong, Z. S., Tan, Y. Y., Chen, S. D., Jin, H. J. \& Yan, Y. (2020). The origin, transmission and clinical therapies on coronavirus disease 2019 (COVID-19) outbreak-an update on the status. Military Medical Research, 7(1), 1-10.

Hamming, I., Timens, W., Bulthuis, M. L. C., Lely, A. T., Navis, G. V., \& van Goor, H. (2004). Tissue distribution of ACE2 protein, the functional receptor for SARS coronavirus. A first step in understanding SARS pathogenesis. The Journal of Pathology: A Journal of the Pathological Society of Great Britain and Ireland, 203(2), 631-637.

Helms, J., Kremer, S., Merdji, H., Clere-Jehl, R., Schenck, M., Kummerlen, C.\& Anheim, M. (2020). Neurologic features in severe SARS-CoV-2 infection. New England Journal of Medicine.

Hoffmann, M., Kleine-Weber, H., Schroeder, S., Krüger, N., Herrler, T., Erichsen, S., \& Müller, M. A. (2020). SARS-CoV-2 cell entry depends on ACE2 and TMPRSS 2 and is blocked by a clinically proven protease inhibitor. Cell.

Huang, C., Wang, Y., Li, X., Ren, L., Zhao, J., Hu, Y.,... \& Cheng, Z. (2020). Clinical features of patients infected with 2019 novel coronavirus in Wuhan, China. The lancet, 395(10223), 497-506.

Imai, Y., Kuba, K., \& Penninger, J. M. (2008). The discovery of angiotensin-converting enzyme 2 and its role in acute lung injury in mice. Experimental physiology, 93(5), 543-548. 
Jacomy, H., Fragoso, G., Almazan, G., Mushynski, W. E., \& Talbot, P. J. (2006). Human coronavirus OC43 infection induces chronic encephalitis leading to disabilities in BALB/C mice. Virology, 349(2), 335-346.

Koyuncu, O. O., Hogue, I. B., \& Enquist, L. W. (2013). Virus infections in the nervous system. Cell host \& microbe, 13(4), 379-393.

Leber, A. L., Everhart, K., Balada-Llasat, J. M., Cullison, J., Daly, J., Holt, S. \& Reed, S. L. (2016). Multicenter evaluation of BioFire FilmArray meningitis/encephalitis panel for detection of bacteria, viruses and yeast in cerebrospinal fluid specimens. Journal of clinical microbiology, 54(9), 2251-2261.

Li, K., Wohlford-Lenane, C., Perlman, S., Zhao, J., Jewell, A. K., Reznikov, L. R. \& McCray Jr, P. B. (2016). Middle East respiratory syndrome coronavirus causes multiple organ damage and lethal disease in mice transgenic for human dipeptidyl peptidase 4 . The Journal of infectious diseases, 213(5), 712-722.

Li, W., Moore, M. J., Vasilieva, N., Sui, J., Wong, S. K., Berne, M. A. \& Choe, H. (2003). Angiotensinconverting enzyme 2 is a functional receptor for the SARS coronavirus. Nature, 426(6965), 450-454.

Li, Y., Wang, M., Zhou, Y., Chang, J., Xian, Y., Mao, L. \& Li, M. (2020a). Acute cerebrovascular disease following COVID-19: a single center, retrospective, observational study.

Li, Z., Wu, M., Yao, J., Guo, J., Liao, X., Song, S. \& Zhou, Z. (2020b). Caution on kidney dysfunctions of COVID-19 patients.

Li, Y. C., Bai, W. Z., \& Hashikawa, T. (2020c). The neuroinvasive potential of SARS-CoV2 may play a role in the respiratory failure of COVID-19 patients. Journal of medical virology, 92(6), 552-555.

Lochhead, J. J., \& Thorne, R. G. (2012). Intranasal delivery of biologics to the central nervous system. Advanced drug delivery reviews, 64(7), 614-628.

Lochhead, J. J., Kellohen, K. L., Ronaldson, P. T., \& Davis, T. P. (2019). Distribution of insulin in trigeminal nerve and brain after intranasal administration. Scientific reports, 9(1), 1-9.

Lu, R., Zhao, X., Li, J., Niu, P., Yang, B., Wu, H.,... \& Bi, Y. (2020). Genomic characterisation and epidemiology of 2019 novel coronavirus: implications for virus origins and receptor binding. The Lancet, 395(10224), 565-574.

Mao, L., Jin, H., Wang, M., Hu, Y., Chen, S., \& He, Q. (2020). Neurologic manifestations of hospitalized patients with coronavirus disease 2019 in Wuhan, China. JAMA Neurol. 2020 Apr.

Mehta, P., McAuley, D. F., Brown, M., Sanchez, E., Tattersall, R. S., Manson, J. J., \& HLH Across Speciality Collaboration. (2020). COVID-19: consider cytokine storm syndromes and immunosuppression. Lancet (London, England), 395(10229), 1033.
Min, C. K., Cheon, S., Ha, N. Y., Sohn, K. M., Kim, Y., Aigerim, A. \& Moon, J. Y. (2016). Comparative and kinetic analysis of viral shedding and immunological responses in MERS patients representing a broad spectrum of disease severity. Scientific reports, 6(1), 1-12.

Netland, J., Meyerholz, D. K., Moore, S., Cassell, M., \& Perlman, S. (2008). Severe acute respiratory syndrome coronavirus infection causes neuronal death in the absence of encephalitis in mice transgenic for human ACE2. Journal of virology, 82(15), 7264-7275.

Osterholm, M. T. (2005). Preparing for the next pandemic. New England Journal of Medicine, 352(18), 1839-1842.

Poyiadji, N., Shahin, G., Noujaim, D., Stone, M., Patel, S., \& Griffith, B. (2020). COVID-19-associated acute hemorrhagic necrotizing encephalopathy: CT and MRI features. Radiology, 201187.

Raj, V. S., Mou, H., Smits, S. L., Dekkers, D. H., Müller, M. A., Dijkman, R. \& Thiel, V. (2013). Dipeptidyl peptidase 4 is a functional receptor for the emerging human coronavirus-EMC. Nature, 495(7440), 251-254.

Reddy, D. S., \& Kuruba, R. (2013). Experimental models of status epilepticus and neuronal injury for evaluation of therapeutic interventions. International journal of molecular sciences, 14(9), 18284-18318.

Reghunathan, R., Jayapal, M., Hsu, L. Y., Chng, H. H., Tai, D., Leung, B. P., \& Melendez, A. J. (2005). Expression profile of immune response genes in patients with severe acute respiratory syndrome. BMC immunology, 6(1), 2.

Rhie, S. J., Jung, E. Y., \& Shim, I. (2020). The role of neuroinflammation on pathogenesis of affective disorders. Journal of Exercise Rehabilitation, 16(1), 2.

Soler, Z. M., Patel, Z. M., Turner, J. H., \& Holbrook, E. H. (2020, January). A primer on viral-associated olfactory loss in the era of COVID-19. In International Forum of Allergy \& Rhinology.

Sun, X., Zhang, X., Chen, X., Chen, L., Deng, C., Zou, X. \& Yu, H. (2020). The infection evidence of SARS-COV-2 in ocular surface: a single-center cross-sectional study. MedRxiv.

Sungnak, W., Huang, N., Bécavin, C., Berg, M., \& Network, H. C. A. (2020). SARS-CoV-2 entry genes are most highly expressed in nasal goblet and ciliated cells within human airways. arXiv preprint arXiv:2003.06122.

Talbot, P. J., Ékandé, S., Cashman, N. R., Mounir, S., \& Stewart, J. N. (1994). Neurotropism of human coronavirus 229E. In Coronaviruses (pp. 339-346). Springer, Boston, MA.

Talbot, P. J., Jacomy, H., \& Desforges, M. (2007). Pathogenesis of human coronaviruses other than severe acute respiratory syndrome coronavirus. Nidoviruses, 313-324. 
Teijaro, J. R., Walsh, K. B., Rice, S., Rosen, H., \& Oldstone, M. B. (2014). Mapping the innate signaling cascade essential for cytokine storm during influenza virus infection. Proceedings of the National Academy of Sciences, 111(10), 3799-3804.

Thompson, R. (2020). Pandemic potential of 2019nCoV. Lancet Infect Dis, 20(3), 280.

Tong, J. Y., Wong, A., Zhu, D., Fastenberg, J. H., \& Tham, T. (2020). The prevalence of olfactory and gustatory dysfunction in COVID-19 patients: a systematic review and meta-analysis. Otolaryngology-Head and Neck Surgery, 0194599820926473.

Vabret, A., Dina, J., Brison, E., Brouard, J., \& Freymuth, F. (2009). Coronavirus humains ( $\mathrm{HCoV})$ Human coronaviruses. Pathol. Biol, 57, 149-160.

Wu, Y., Xu, X., Chen, Z., Duan, J., Hashimoto, K., Yang, L. \& Yang, C. (2020). Nervous system involvement after infection with COVID-19 and other coronaviruses. Brain, behavior and immunity.

Xia, H., \& Lazartigues, E. (2010). Angiotensinconverting enzyme 2: central regulator for cardiovascular function. Current hypertension reports, 12(3), 170-175.

Xiang, P., Xu, X. M., Gao, L. L., Wang, H. Z., Xiong, H. F., \& Li, R. H. (2020). First case of 2019 novel coronavirus disease with encephalitis. ChinaXiv, 202003, 00015.

Xu, J., Zhong, S., Liu, J., Li, L., Li, Y., Wu, X.,... \& Ding, Y. (2005). Detection of severe acute respiratory syndrome coronavirus in the brain: potential role of the chemokine mig in pathogenesis. Clinical infectious diseases, 41(8), 1089-1096.
Xu, Z., Shi, L., Wang, Y., Zhang, J., Huang, L., Zhang, C. \& Tai, Y. (2020). Pathological findings of COVID-19 associated with acute respiratory distress syndrome. The Lancet respiratory medicine, 8(4), 420-422.

Zhang, L., Shen, F. M., Chen, F., \& Lin, Z. (2020 February). Posting date Origin and evolution of the 2019 novel coronavirus. Clin Infect Dis doi, 10.

Zhao, H., Shen, D., Zhou, H., Liu, J., \& Chen, S. (2020). Guillain-Barré syndrome associated with SARSCoV-2 infection: causality or coincidence? The Lancet Neurology, 19(5), 383-384.

\section{Abbreviations used in this Study}

CNS: $\quad$ Central Nervous System

PNS: $\quad$ Peripheral Nervous System

SARS-CoV-2: Severe Acute Respiratory Syndrome Coronavirus 2

ACE-2: $\quad$ Angiotensin Converting Enzyme 2

CoVs: $\quad$ Coronaviruses

BBB: $\quad$ Blood Brain Barrier

MERS-CoV: Middle East Respiratory Syndrome Coronavirus

DPP4: $\quad$ Dipeptidyl-Peptidase 4

ROS: $\quad$ Reactive Oxygen Species

DVC: Dorsal Vagal Complex

GBS: Guillain Barré Syndrome

MS: $\quad$ Multiple Sclerosis

IL: $\quad$ Interleukin 